\title{
A Note about Total Stability of a Class of Hybrid Systems
}

\author{
Manuel De la SEN \\ Departamento de Electricidad y Electronica \\ Instituto de Investigación y Desarrollo de Procesos IIDP \\ Facultad de Ciencias, Universidad del País Vasco \\ Campus de Leioa (Bizkaia), Aptdo. 644 de Bilbao, 48080-Bilbao, Spain \\ e-mail:msen@we.lc.ehu.es
}

Received: December 2005

\begin{abstract}
Robust stability results for nominally linear hybrid systems are obtained from total stability theorems for purely continuous-time and discrete-time systems. The class of hybrid systems dealt with consists of, in general, coupled continuous-time and digital systems subject to state perturbations whose nominal (i.e., unperturbed) parts are linear and time-varying, in general. The obtained sufficient conditions on robust stability are dependent on the values of the parameters defining the over-bounding functions of the uncertainties and the weakness of the coupling between the analog and digital sub-states provided that the corresponding uncoupled nominal subsystems are both exponentially stable.
\end{abstract}

Key words: dynamic hybrid systems, stability, total stability.

\section{Introduction}

Stability of both continuous-time and discrete-time singularly perturbed systems has received much attention in the last years (Kafri and Abed, 1996; Kolev, 1993; Shieh et al., 1986; Oppenheimer and Michel, 1988). Also, stability analysis of discrete-time singularly perturbed systems with calculations of parameter bounds has been reported in (Kolev, 1993; Shieh et al., 1986). An assumption used in previous work to carry out the stability analysis of singularly perturbed systems is relaxed in (Kafri and Abed, 1996) where an upper-bound on the singular perturbation parameters is included to derive such an analysis. On the other hand, the so-called hybrid models are a very important tool for analysis in the modern computers and control technologies since they describe usual situations where continuous-time and either discrete-time and /or digital systems are coupled (De la Sen, 1996; Kabamba and Hara, 1993). A usual example, very common in practice, is the case when a digital controller operates over a continuous-time plant to stabilize it or to improve its performance. The systems described in (De la Sen, 1996; Kabamba and Hara, 1993) are more general since the controlled plant can also possess an hybrid nature since all the continuous-time and digital state- variables can be mutually coupled and to possess internal delays (De la Sen and Alastruey, 2004; De la Sen, 2004; De la Sen and 
Luo, 2004). Other studies and development concerned with hybrid systems in the contexts of presence of delays in their dynamics, fundamental properties of dynamic systems and manufacturing systems have been recently performed in (De la Sen, 2006; Bargelis et al., 2004; Chaib et al., 2005; Marchenko and Poddubnaya, 2005). In this brief paper, stability results are obtained for a wide class of such systems whose nominal (i.e., unperturbed) parts are linear and, in general, time-varying while the state perturbations are allowed to be, in general, non-linear, time-varying and of a dynamic nature. The results about robust stability are obtained by firstly deriving sufficient stability conditions related to total stability for an extended discrete system which describes the overall state trajectory at sampling instants via the discretization of the continuous-time sub-state. Subsequently, a result about total stability of the continuous-time sub-state is carried out to ensure the system' s stability during the inter-sample intervals. Some links with the results given in (Kafri and Abed, 1996) about singularly perturbed systems are also given for a special hybrid system within the given class.

Notation. $\lambda_{\max }(M)$ and $\operatorname{det}(M)$ denote, respectively the maximum eigenvalue and determinant of the square matrix $M=\left(M^{(i j)}\right)$. The symbol $\otimes$ denotes the direct Kronecker product of matrices. Particular norms for functions, sequences or matrices are denoted by the appropriate subscript. In the expressions being valid for any norms, those subscripts are omitted.

\section{Problem Statement}

\section{A) Plant Description $\Sigma$}

Consider the following, in general, time-varying hybrid plant

System $\Sigma$ :

$$
\begin{aligned}
& \dot{x}_{c}(t)=A_{c}(t) x_{c}(t)+A_{c d}(t) x_{d}[k]+\delta_{c}(t), \\
& x_{d}[k+1]=A_{d}[k] x_{d}[k]+A_{d c}[k] x_{c}[k]+\delta_{d}[k], \\
& \delta_{c}(t)=f_{c c}\left(t, x_{c}(t)\right)+f_{c d}\left(t, x_{d}[k]\right)+g_{c c}\left(t, x_{c}(t)\right)+g_{c d}\left(t, x_{d}[k]\right), \\
& \delta_{d}[k]=f_{d c}\left(k, x_{c}[k]\right)+f_{d d}\left(k, x_{d}[k]\right)+g_{d c}\left(k, x_{c}[k]\right)+g_{d d}\left(k, x_{d}[k]\right)
\end{aligned}
$$

for all time $t \in[k T,(k+1) T)$ and discrete time integer index $k \geqslant 0$ for sampling period $T$ where $x_{c}(t)$ and $x_{d}[k]$ are, respectively, the $n_{c}$ continuous-time (or analog) substate and $n_{d}$ discrete-time (or digital) substate. The continuous-time and discrete-time variables are denoted by $(t)$ and $[k]$, respectively. The discretized analog substate at sampling instants is denoted as a digital signal; i.e., $x_{c}(k T)=x_{c}[k]$. The matrix functions $A_{c}(t), A_{c d}(t)$, $A_{d}[k]$ and $A_{d c}[k]$ are of dimensions being compatible with the corresponding vectors in $(1)-(2) . \delta_{c}(t)$ and $\delta_{d}[k]$ are disturbances being, in general, nonlinear and time-varying subject to the following set of constraints on the functions $f_{(.)}$and $g_{(.)}$.

Constraints $C$ :

C1) $A_{d}[k], A_{d c}[k], f_{d c}\left(k, x_{c}[k]\right), f_{d d}\left(k, x_{d}[k]\right), g_{d c}\left(k, x_{c}(t)\right), g_{d d}\left(k, x_{d}[k]\right)$ matrix and vector sequences of $k$ of bounded entries. The entries of $A_{c}(t), A_{c d}(t), f_{c c}\left(t, x_{c}(t)\right)$, 
$f_{c d}\left(t, x_{d}[k]\right), g_{c c}\left(t, x_{c}(t)\right)$ and $g_{c d}\left(t, x_{d}[k]\right)$ are locally integrable functions of $t$ for each fixed $x$ in the ball $\operatorname{Max}\left(\left\|x_{c}\right\| \leqslant r,\left\|x_{d}\right\| \leqslant r\right)$ and all integer $k \geqslant 0$ and all $t \geqslant 0$.

$$
\begin{array}{ll}
\text { C2) } & f_{c c}(t, 0)=f_{d c}(t, 0)=0 \in \mathbf{R}^{n_{c}}, \quad f_{d c}(k, 0)=f_{d d}(k, 0)=0 \in \mathbf{R}^{n_{d}}, \\
\text { C3) } & \left\|h_{c c}\left(t, x_{c 1}\right)-h_{c c}\left(t, x_{c 2}\right)\right\| \leqslant \beta_{c c}^{h}\left\|x_{c 1}-x_{c 2}\right\|, \\
& \left\|h_{c d}\left(t, x_{c 1}\right)-h_{c d}\left(t, x_{c 2}\right)\right\| \leqslant \beta_{c d}^{h}\left\|x_{c 1}-x_{c 2}\right\|, \\
& \left\|h_{d c}\left(k, x_{d 1}\right)-h_{d c}\left(k, x_{d 2}\right)\right\| \leqslant \beta_{d c}^{h}\left\|x_{d 1}-x_{d 2}\right\|, \\
& \left\|h_{d d}\left(k, x_{d 1}\right)-h_{d d}\left(k, x_{d 2}\right)\right\| \leqslant \beta_{d d}^{h}\left\|x_{d 1}-x_{d 2}\right\|, \\
\text { C4) } & \left\|g_{c c}\left(t, x_{c 1}\right)\right\| \leqslant \beta_{c c}^{g} r, \quad\left\|g_{c d}\left(t, x_{d 1}\right)\right\| \leqslant \beta_{c d}^{g} r, \\
& \left\|g_{d c}\left(t, x_{c 1}\right)\right\| \leqslant \beta_{d c}^{g} r, \quad\left\|g_{d d}\left(t, x_{d 1}\right)\right\| \leqslant \beta_{d d}^{g} r,
\end{array}
$$

for all $\left\|x_{c i}\right\| \leqslant r,\left\|x_{d i}\right\| \leqslant r$ and all integer $k \geqslant 0$ and all $t \geqslant 0$, with $\mathrm{h}$ being any of the vector real functions or sequences of (3)-(4) and $\beta_{(.)}^{h}(h=f$ or $h=g$ ) being known nonnegative real constants. The problem dealt with in this brief is the investigation of the robust stability of $\Sigma$ (i.e., that of (1)-(2) with dynamic state disturbances (3)-(4)) subject to the set of constraints $C$. For this purpose, the state-trajectory of $\Sigma$ at sampling instants is calculated in the following subsection.

B) Extended Discrete System $\Sigma_{d}$

Direct calculation of the solution of $\Sigma$ at sampling instants (i.e., $t=k T, k$ being any nonnegative integer) yields the following discrete extended system:

$$
\Sigma_{d}: x[k+1]=A[k] x[k]+\delta[k], \quad \text { all integer } k \geqslant 0
$$

with $x[k]=\left(x_{c}^{T}[k], x_{d}^{T}[k]\right)^{T}$ subject to $x[0]=\left(x_{c}^{T}[0], x_{d}^{T}[0]\right)^{T}$, with $x_{c}[0]=x_{c}(0)$, and

$$
\begin{aligned}
& A[k]=\left[\begin{array}{ll}
\Phi_{c}[k] & \Gamma_{c}[k] \\
A_{d c}[k] & A_{d}[k]
\end{array}\right], \quad \Gamma_{c}[k]=\int_{0}^{T} \Phi_{c}((k+1) T-\tau) A_{c d}(k T+\tau) \mathrm{d} \tau, \\
& \delta[k]=\left(\delta_{c}^{\prime} T(t), \delta_{d}^{T}[k]\right)=\left(\int_{0}^{T} \delta_{c}^{T}(k T+\tau) \Phi_{c}^{T}((k+1) T-\tau) \mathrm{d} \tau, \delta_{d}^{T}[k]\right)^{T},
\end{aligned}
$$

$\Phi_{c}(t)$ and $\Gamma_{c}(t)$ being defined at sampling instants as $\Phi_{c}[k]=\Phi_{c}(k T)=\Psi_{c}((k+1) T, k T)$ and $\Gamma_{c}[k]=\Gamma_{c}(k T)$ are the $k$-th intersample state transition and control matrices of the continuous subsystem, respectively, (i.e., $\dot{\Psi}_{c}(t, 0)=A_{c}(t) \Psi_{c}(t, 0) ; \Psi_{c}(0,0)=I_{n_{c}}$ for all $t \in[k T,(k+1) T)$ and all integer $k \geqslant 0$.

\section{Main Results}

The robust stability of $\Sigma$ subject to the constraints $C$ under the knowledge of the constants $\beta_{(.)}^{(.)}$is now investigated. The results on robust stability are useful for both local and global stability in the sense that stability is ensured for initial conditions of (1)-(4) being constrained to the balls $\left\|x_{c}(0)\right\| \leqslant r,\left\|x_{d}[0]\right\| \leqslant r$ where the radius $r$ is arbitrary but compatible with the validity of the constraints $C$ on $\Sigma$. 
A) Exponential Stability of the Nominal Extended System $\Sigma_{d}^{*}$

The nominal $\Sigma$ is defined by zeroing $\delta_{c}(t)$ and $\delta_{d}[k]$ in (1)-(2). This results into the nominal version $\Sigma_{d}^{*}$ of $\Sigma_{d}$ in (10)-(12) satisfying $x^{*}[k+1]=A[k] x^{*}[k]$ with $x^{*}[0]=$ $\left(x_{c}^{T}[0], x_{d}^{T}[0]\right)^{T}$. The following assumption is given:

AsSUMPTION 1. The nominal uncoupled continuous-time and digital subsystems $\dot{x}_{c}^{*}(t)=A_{c}(t) x_{c}^{*}(t)$ and $x_{d}^{*}[k+1]=A_{d}[k] x_{d}^{*}[k]$ are both exponentially stable, i.e., thee exist norm-dependent real constants $K_{c} \geqslant 1$ and $K_{d} \geqslant 1$ such that $\left\|\Psi_{c}\left(t_{2}, t_{1}\right)\right\| \leqslant$ $K_{c} \mathrm{e}^{-a_{c}\left(t_{2}-t_{1}\right)}$ and $\left\|\Psi_{d}\left(k_{2}, k_{1}\right)\right\| \leqslant K_{d} a_{d}^{k_{2}-k_{1}}$ for some real constants $a_{c}>0$ and $a_{d} \in[0,1)$ where $\Psi_{c}(.,$.$) and \Psi_{d}[.,$.$] are the state-transition matrices of the un-$ coupled continuous-time and digital subsystems in $\Sigma$ (i.e., $\dot{\Psi}_{c}(t, 0)=A_{c}(t) \Psi_{c}(t, 0)$; $\Psi_{c}(0,0)=I_{n_{c}}$ with $\Psi_{c}\left(k_{2} T, k_{1} T\right)=\prod_{j=k_{1}}^{j=k_{2}-1} \Phi[j]$ between two sampling instants and $\Psi_{d}\left[k_{2}, k_{1}\right]=\prod_{j=k_{1}}^{j=k_{2}-1} A_{d}[j]$ with $\Psi_{d}[0,0]=I_{n_{d}}$ for all $t \geqslant 0$, any real $t_{2} \geqslant t_{1} \geqslant 0$ and any integers $k_{2} \geqslant k_{1} \geqslant 0$ ).

The following stability result holds for the nominal extended system (i.e., $\delta \equiv 0$ in (10)).

Proposition 1. Define

$$
\begin{gathered}
\rho_{k}=\operatorname{Max}\left(\operatorname{Max}_{1 \leqslant i \leqslant n_{c}}\left(\sum_{j=1}^{n_{c}} \int_{0}^{T}\left|\Psi_{c}^{(i j)}((k+1) T, k T+\tau) A_{c d}(\tau) \mathrm{d} \tau\right|\right),\right. \\
\left.\underset{1 \leqslant i \leqslant n_{d}}{\operatorname{Max}}\left(\sum_{j=1}^{n_{d}}\left|A_{d c}^{(i j)}[k]\right|\right)\right) .
\end{gathered}
$$

Thus, the nominal extended discrete system is exponentially stable if Assumption 1 holds and $\rho_{k}<1-\operatorname{Max}\left(\mathrm{e}^{-a_{c} T}, a_{d}\right)$ for all integer $k \geqslant 0$.

Proof. Decompose $A[k]=A_{0}[k]+\widetilde{A}[k]$ in (11) with $A_{0}[k]=\operatorname{Block} \operatorname{Diag}\left(\Phi_{c}[k], A_{d}[k]\right)$, $\Phi_{c}[k]=\Psi_{c}((k+1) T, k T)$ and $A_{d}[k]=\Psi_{d}[(k+1) T, k]$ being the one sampling period $k$ th transition matrices. Thus, $x^{*}[k+1]=A[k] x^{*}[k]$ is exponentially stable if there exist real constants $\bar{K} \geqslant 1$ (being norm-dependent) and $\bar{a} \in[0,1)$ such that its state transition matrix $\Psi\left[k_{2}, k_{1}\right]=\prod_{j=k_{1}}^{k_{2}-1} A[j]$ satisfies $\left\|\Psi\left[k_{2}, k_{1}\right]\right\| \leqslant \bar{K} \bar{a}^{k_{2}-k_{1}} \bar{K}=1$ for the $l_{2}$-matrix norm given by the maximum modulus within the whole set of eigenvalues. Also, $\rho_{k}=\|\widetilde{A}[k]\|_{2}$, from the definition of $\rho_{k}$ and $\widetilde{A}[k]=A[k]-A_{0}[k]$, lies in the union $\cup_{i=1}^{n_{c}} R_{i}$ of the discs $R_{i}=\left\{z:|z| \leqslant \sum_{j=1}^{n_{c}+n_{d}}\left|\widetilde{A}^{(i j)}[k]\right|\right\}$ from Gerschgorin's circle theorem (Kincaid and Cheney, 1991). Therefore, $\|A[k]\|_{2} \leqslant \operatorname{Max}\left(\mathrm{e}^{-a_{c} T}, a_{d}\right)+\rho_{k} \leqslant$ $\bar{a}<1$ for all integer $k \geqslant 0$ if Assumption 1 and (13) hold. Thus, the nominal extended system is exponentially stable and the result has been proved.

B) Stability of the Discrete Disturbed Extended System $\Sigma_{d}$

The following result gives sufficient conditions for stability of the extended discrete system (10) within a closed ball of the extended state $x[$.$] .$ 
Proposition 2. Assume that Proposition 1 holds under Assumption 1 (i.e., the nominal extended system (10) is exponentially stable) under the stronger condition $\|A[k]\|_{2} \leqslant$ $\bar{a}<1-\frac{\bar{K}_{d}}{\bar{K}}<1$ where the real constants $\bar{K}$ and $\bar{a}$ are related to the state transition matrix of $\Sigma_{d}$ Eq. 10 and defined in the proof of Proposition 1, and

$$
\begin{aligned}
& \bar{K}_{d}=K_{c} a_{c}^{-1} \beta_{c}+K_{d} \beta_{d}, \\
& \beta_{h}=\beta_{h c}^{f}+\beta_{h d}^{f}+\beta_{h c}^{g}+\beta_{h d}^{g}
\end{aligned}
$$

for $h=c, d$; and $\bar{K}_{d}<1$. Thus, the state vector is uniformly bounded according to

$$
\|x[k]\| \leqslant\left(\bar{a}^{k}+\bar{K} \bar{K}_{d} \frac{1-\bar{a}^{k}}{1-\bar{a}}\right) r \leqslant r
$$

for all integer $k \geqslant 0$ provided that $\operatorname{Max}\left(\left\|x_{c}[0]\right\|,\left\|x_{d}[0]\right\|\right)<\frac{r}{2 \bar{K}} \leqslant \frac{r}{2}$.

Proof. First, note from direct calculus from (6)-(9) that the disturbance signal $\delta[k]$ in (10) satisfies

$$
\|\delta[k]\| \leqslant\left\|\delta_{c}^{\prime}[k]\right\|+\left\|\delta_{d}[k]\right\| \leqslant \bar{K}_{d} r
$$

provided that $\operatorname{Max}\left(\left\|x_{c}[k]\right\|,\left\|x_{d}[k]\right\|\right)<r / 2$ for all integer $k \geqslant 0$ since $\bar{K}>1$ and $\bar{a}<1-\frac{\bar{K}_{d}}{\bar{K}}$ imply $\bar{a}+\bar{K}_{d}<1$. Consider the set of sequences $\{y[k], k \geqslant 0\}$ equipped with the $\ell_{\infty}$ norm for sequences $\|y\|_{\infty}=\operatorname{Max}_{0 \leqslant k \leqslant \infty}(\|y[k]\|)$. Thus, the operator $\mathbf{T}_{\mathbf{d}}$ defined by $\left(\mathbf{T}_{\mathbf{d}} y\right)[k]=A[k] y[k]+\delta[k]$ is a contraction on the closed subset $\mathbf{R}_{\mathbf{d}}$ of bounded $n_{d^{-}}$ vector sequences $\left\{y[k], k \geqslant 0:\|y\|_{\infty} \leqslant r\right\}$. By the contraction mapping theorem (Hale, 1980; Kincaid and Cheney, 1991) there is a unique solution $y[k+1]=\left(\mathbf{T}_{\mathbf{d}} y\right)[k]$ (fixed point) with sequences in $\mathbf{R}_{\mathbf{d}}$, and

$$
\|x[k]\|=\left\|\Psi[k, 0] x[0]+\sum_{i=0}^{k-1} \Psi[k, i+1] \delta[i]\right\| \leqslant \bar{K} \bar{a}^{k}\|x[k]\|+\bar{K} \bar{K}_{d} r \sum_{i=0}^{k-1} \bar{a}^{i}
$$

which leads directly to (16) since $\bar{a}<1-\frac{\bar{K}_{d}}{\bar{K}}<1$ implies

$$
\sum_{i=0}^{k-1} \bar{a}^{i}=\frac{1-\bar{a}^{k}}{1-\bar{a}} ; \quad \bar{a}^{k}+\bar{K} \bar{K}_{d} \frac{1-\bar{a}^{k}}{1-\bar{a}} \leqslant 1+\bar{a}^{k}-\bar{a} \leqslant 1 .
$$

C) Stability of the Continuous-Time Substate Inbetween Sampling Instants

Now, the solution to (1) subject to (2)-(3) is analyzed by taking into account that $\|x[k]\| \leqslant r$ provided that Proposition 2 holds. A total stability argument is used as main tool for the proof of stability of the continuous-time subsystem.

Proposition 3. Assume that Proposition 2 holds, $\operatorname{Sup}_{0 \leqslant t<\infty}\left(\left\|A_{c d}(t)\right\|\right) \leqslant a_{c d}$, $\left\|x_{c}(0)\right\| \leqslant \frac{r}{2 K_{c}}$ and $\frac{K_{c} \bar{K}_{c}}{a_{c}}<1$, where

$$
\bar{K}_{c}=K_{c}^{f}+K_{c}^{g} ; \quad K_{c}^{f}=\beta_{c c}^{f}+\beta_{c d}^{f} ; \quad K_{c}^{g}=a_{c d}+\beta_{c c}^{f}+\beta_{c d}^{g} .
$$


Thus, there is a unique solution $x_{c}(t)$ to (1) such that for all $t \geqslant 0$ :

$$
\left\|x_{c}(t)\right\| \leqslant K_{c} \mathrm{e}^{-\left(a_{c}-K_{c} K_{c}^{f}\right) t}\left\|x_{0}\right\|+\frac{K_{c} K_{c}^{g}}{a_{c}-K_{c} K_{c}^{f}} r\left(1-\mathrm{e}^{-\left(a_{c}-K_{c} K_{c}^{f}\right) t}\right) \leqslant r .
$$

Proof. One gets directly from (1),

$$
x_{c}(t)=\Psi_{c}(t, 0) x_{c}(0)+\int_{0}^{t} \Psi_{c}(t-\tau) \delta_{c}^{0}(\tau) \mathrm{d} \tau
$$

with $x_{c}(0)=x_{c}[0]$ and $\delta_{c}^{0}(t)=A_{c d}(t) x_{d}[k]+\delta_{c}(t)$. Under the set of constraints $C,\left\|\delta_{c}^{0}(t)\right\| \leqslant \bar{K}_{c} r$ for all $t \geqslant 0$ subject to (20). Using similar arguments as in the proof of Proposition 2, consider the Banach space $\mathbf{B}_{\mathbf{c}}=C[0, \infty)$ of continuous, bounded $n_{c}$-vector sequences defined on $[0, \infty)$ and equipped with the $L_{\infty}$-norm $\|y\|_{\infty}=\operatorname{Sup}_{0 \leqslant t \leqslant \infty}(|y(t)|)$. The operator $\mathbf{T}_{\mathbf{c}}$ is defined via

$$
\left(\mathbf{T}_{\mathbf{c}} y\right)(t)=\Psi_{c}(t, 0) x_{0}+\int_{0}^{t} \Psi_{c}(t, \tau) \delta_{c}^{0}(\tau) \mathrm{d} \tau
$$

is a contraction of the closed subset $\mathbf{R}_{\mathbf{c}}=\left\{y \in \varepsilon \mathbf{B}_{\mathbf{c}}:\|y\|_{\infty} \leqslant r\right\}$ of $\mathbf{B}_{\mathbf{c}}$, because for $\left\|y_{i}\right\|_{\infty} \leqslant r(i=1,2)$, one gets from (20)and (23)

$$
\begin{gathered}
\left|\left(\mathbf{T}_{\mathbf{c}} y\right)(t)\right| \leqslant K_{c}\left\{\mathrm{e}^{-a_{c} t}\left\|x_{c}(0)\right\|+\frac{\bar{K}_{c}}{a_{c}}\left(1-\mathrm{e}^{-a_{c} t}\right) r\right\} \leqslant r \\
\Rightarrow\left\|\mathbf{T}_{\mathbf{c}} y_{1}-\mathbf{T}_{\mathbf{c}} y_{2}\right\| \leqslant \bar{K}_{c} a_{c}^{-1}\left\|y_{1}-y_{2}\right\| \leqslant\left\|y_{1}-y_{2}\right\|
\end{gathered}
$$

for $\left\|x_{c}(0)\right\|=\left\|x_{c}[0]\right\| \leqslant \frac{r}{2 K_{c}} \leqslant \frac{r}{2 \bar{K}} \leqslant r$ since $\left\|x_{d}[k]\right\| \leqslant \frac{r}{2 \bar{K}} \leqslant r$ for all $k \geqslant 0$ from Proposition 2. By the contraction mapping theorem, (Hale, 1980; Kincaid and Cheney, 1991), there exists a unique solution of (23) in $\mathbf{R}_{\mathbf{c}}$, the fixed point of $\mathbf{T}_{\mathbf{c}}$. Thus, one gets from (23) that

$$
\|x(t)\| \leqslant K_{c}\left\{\mathrm{e}^{-a_{c} t}\left\|x_{0}\right\|+K_{c}^{f} \int_{0}^{t} \mathrm{e}^{-a_{c}(t-\tau)}\left\|x_{c}(\tau)\right\| \mathrm{d} \tau+K_{c}^{g} r \int_{0}^{t} \mathrm{e}^{-a_{c}(t-\tau)} \mathrm{d} \tau\right\}
$$

which leads to (21) from Bellman-Gronwall Lemma (Hale, 1980).

REMARK 1 (combined interpretation of Propositions 1-3). Assumption 1 and Propositions $1-3$ yield the following robust stability conditions for the system $\Sigma$ by using $l_{2}$ vector and matrix norms, i.e., $K_{c}=K_{d}=1$, provided that $\left\|x_{c}[0]\right\| \leqslant \frac{r}{2}$ and $\left\|x_{d}[0]\right\| \leqslant \frac{r}{2}$ :

$$
\begin{aligned}
& \rho^{*}+\rho+\beta_{c} a_{c}^{-1}+\beta_{d}<1, \quad \bar{K}_{c} a_{c}^{-1}<1, \\
& \rho^{*}=\operatorname{Max}_{0 \leqslant k \leqslant \infty}\left(\mathrm{e}^{-a_{c} T}, a_{d}\right), \quad \rho=\operatorname{Max}_{0 \leqslant k \leqslant \infty}\left(\rho_{k}\right)
\end{aligned}
$$

with $\beta_{c}$ and $\beta_{d}$ being real constants defined in (15) related to the set of constraints $C, \rho_{k}$ and $\bar{K}_{c}$ defined in (12) and (20). In particular: (a) $\rho^{*}<1$ guarantees the exponential 
stability of the uncoupled nominal continuous-time and digital subsystems (i.e., $\delta_{c} \equiv 0$, $\delta_{d} \equiv 0$ ). (b) $\rho^{*}+\rho<1$ guarantees that the exponential stability is not destroyed in the nominal extended system $\Sigma_{d}^{*}$ by the existence of linear couplings between the continuoustime and digital substates. (c) The first inequality in (26) guarantees that the state disturbances in $\Sigma$ are sufficiently small in terms of the real constants defining their overbounding functions while satisfying $C$ so that the extended discrete system $\Sigma_{d}$ maintains the stability of its nominal description $\Sigma_{d}^{*}$. If, furthermore, the second constraint of (26) holds then the signal boundedness is kept in-between sampling instants according to (21) and the overall hybrid system $\Sigma$ is robustly stable.

\section{D) Links With Singular Perturbation Theory}

In some particular descriptions within the class $\Sigma$, the perturbation theory can be combined with the above analysis. Assume, for instance that the linear dynamics of $\Sigma$ is subject to variations defined by a small parameter $\varepsilon, A_{d c}$ and $A_{d d}$ are time-invariant and $A_{c}(t)=\varepsilon A_{c}$ for all $t \geqslant 0$ and $A_{c d}(t)=\rho(\varepsilon) \mathrm{e}^{\varepsilon A_{c} t}$ with $\rho(\varepsilon) \leqslant \bar{\rho}<\infty$ for all $\varepsilon \in\left[0, \varepsilon^{*}\right)$. Thus, a direct series expansion around $\varepsilon T$ of the state transition matrix of the continuous subsystem yields

$$
\begin{aligned}
& \Psi_{c}((k+1) T, k T)=\mathrm{e}^{\varepsilon A_{c} T} \\
& \quad=I_{n_{c}}+\varepsilon A_{c} T+\Delta\left(\varepsilon, T, A_{c}\right) \int_{k T}^{(k+1) T} \Psi_{c}((k+1) T) A_{c d}(\tau) \mathrm{d} \tau \\
& \quad=\left(I_{n_{c}}+\varepsilon A_{c} T+\Delta\left(\varepsilon, A_{c}, T\right)\right) \int_{k T}^{(k+1) T} \mathrm{e}^{-\varepsilon A_{c} \tau} A_{c d}(\tau) \mathrm{d} \tau \\
& \quad=\left(\varepsilon A_{c}+\rho(\varepsilon)\left[I_{n_{c}}+\Delta\left(\varepsilon, T, A_{c}\right)\right]\right) T .
\end{aligned}
$$

Note that $\left\|\Delta\left(\varepsilon, T, A_{c}\right)\right\|_{2} \leqslant 1+\varepsilon A_{c} T+\varepsilon T\left|\lambda_{\max }\left(A_{c}\right)\right|=\delta(\varepsilon) \leqslant \bar{\delta}<\infty$ for all $\varepsilon \in\left[0, \varepsilon^{*}\right)$ and $\rho(\varepsilon) T\left[I_{n_{c}}+\Delta\left(\varepsilon, T, A_{c}\right)\right] \leqslant \bar{\rho} T(1+\bar{\delta})<\infty$ for all $\varepsilon \in\left[0, \varepsilon^{*}\right)$. Thus,

$$
\begin{aligned}
& A(\varepsilon)=A^{*}(\varepsilon)+\Delta \bar{A}(\varepsilon), \\
& A^{*}(\varepsilon)=\left[\begin{array}{cc}
I_{n_{c}}+\varepsilon A_{c} T & \varepsilon A_{c} T \\
A_{d c} & A_{d}
\end{array}\right], \\
& \Delta \bar{A}(\varepsilon)=\left[\begin{array}{cc}
\Delta\left(\varepsilon, T, A_{c}\right) & \rho(\varepsilon) T\left(I_{n_{c}}+\Delta\left(\varepsilon, T, A_{c}\right)\right) \\
0 & 0
\end{array}\right]
\end{aligned}
$$

is time-invariant in (10). Thus, the discrete system $\Sigma_{d}$ of (10) satisfies equivalently,

$$
z[k+1]=\widehat{A}^{*}(\varepsilon) z[k]+(\delta[k]+\Delta \bar{A}(\varepsilon) z[k])
$$

by defining (see (Kafri and Abed, 1996))

$$
\widehat{A}^{*}(\varepsilon)=\hat{I}+\widehat{A}_{21}+\widehat{A}_{22}+\varepsilon\left(\widehat{A}_{11}+\widehat{A}_{12}\right) \text { for } \varepsilon \in\left[0, \varepsilon^{*}\right)
$$


through the extended $n=n_{c}+n_{d}$-matrices

$$
\begin{array}{ll}
\widehat{A}_{11}=\left[\begin{array}{cc}
T A_{c} & 0 \\
0 & 0
\end{array}\right], & \widehat{A}_{12}=\left[\begin{array}{cc}
0 & T A_{c} \\
0 & 0
\end{array}\right], \quad \hat{I}=\left[\begin{array}{cc}
I_{n_{c}} & 0 \\
0 & 0
\end{array}\right], \\
\widehat{A}_{21}=\left[\begin{array}{cc}
0 & 0 \\
A_{d c} & 0
\end{array}\right], & \widehat{A}_{22}=\left[\begin{array}{cc}
0 & 0 \\
0 & A_{d}
\end{array}\right] .
\end{array}
$$

Note that Schur's stability of $\widehat{A}^{*}(\varepsilon)$ is equivalent to exponential stability of the unforced time-invariant system $\Sigma_{d}^{*}: z^{*}[k+1]=\widehat{A}^{*}(\varepsilon) z^{*}[k]$ since $\widehat{A}^{*}(\varepsilon)$ has its eigenvalues in $|z|<1$ for all $\varepsilon \in\left[0, \varepsilon^{*}\right)$. Thus, the subsequent result follows directly from Proposition 2 by using a previous result in (Kafri and Abed, 1996):

PRoposition 4. Define $\nu(\varepsilon):=\nu(\widehat{A}(\varepsilon))=\operatorname{det}(\widehat{A}(\varepsilon) \otimes \widehat{A}(\varepsilon)-\hat{I} \otimes \hat{I})$ where $\otimes$ denotes the direct Kronecker product of matrices which is a matrix of dimension $(n+m)^{2} \times(n+$ $m)^{2}$. Thus, the following items hold:

(i) If $\nu(\varepsilon)$ has no positive zeros, then either $\widehat{A}(\varepsilon)$ is Schur stable for all $\varepsilon>0$ or it is not Schur stable for any $\varepsilon>0$.

(ii) If $\nu(\varepsilon)$ has positive zeros, let $\bar{\varepsilon}$ be the smallest such zero. If $\widehat{A}\left(\varepsilon_{1}\right)$ is Schur stable for any $\varepsilon_{1} \in(0, \bar{\varepsilon})$ then $\varepsilon^{*}=\bar{\varepsilon}$. Otherwise, $\widehat{A}(\varepsilon)$ is not Schur stable for all sufficiently small and positive values of $\varepsilon$.

(iii) The extended discrete system $\Sigma_{d}(\varepsilon)$ is stable for all $\varepsilon \in\left[0, \varepsilon^{*}\right)$ satisfying $\operatorname{Max}\left(\left\|x_{c}[0], x_{d}[0]\right\|\right) \leqslant \frac{r}{2 \bar{K}}$ and $\left\|A^{*}(\varepsilon)\right\|_{2}+\bar{K}_{d}+\operatorname{Max}_{0 \leqslant \varepsilon \leqslant \varepsilon^{*}}\left(\|\Delta A(\varepsilon)\|_{2}\right)<1$ with $\bar{K}_{d}$ defined in (14).

\section{Simulated Example}

The following third-order system, whose state-space description lie within the class of hybrid plants (1), is considered:

$$
\begin{aligned}
& \ddot{y}(t)+a_{1} \dot{y}(t)+a_{2} y(t)+a_{3} y[k]+4 y[k-1] \\
& \quad=b_{0} u(t)+b_{1} \dot{u}(t)+b_{2} u[k]+3 u[k-1]+0.3(z[k]+\delta(t)), \\
& z[k+1]=0.2 z[k]+1.1 u[k]+1.3 y[k], \quad \dot{\delta}(t)=-7 \delta(t)+8.5 u(t)
\end{aligned}
$$

for all $t \in[k T,(k+1) T)$ and any integer $k \geqslant 0$. The signal $u(t)$ is a stabilizing outputfeedback control signal generated from an hybrid controller as follows:

$$
\begin{aligned}
& u(t)=\frac{G_{1}(D, q)}{L(D, q)} u(t)+\frac{G_{2}(D, q)}{L(D, q)} y(t), \\
& G_{1}(D, q)=D^{2} q^{2}-q^{2} D+D^{2} q+1.25 q^{2}-D q+0.25 q \\
& \quad-1.44187 D^{2}+0.206426 D-2.54251, \\
& G_{2}(D, q)=1.12792\left(D^{2} q^{2}-0.269774 q^{2} D+1.10629\right), \\
& L(D, q)=(D-0.5)^{2}(q+0.5)^{2},
\end{aligned}
$$




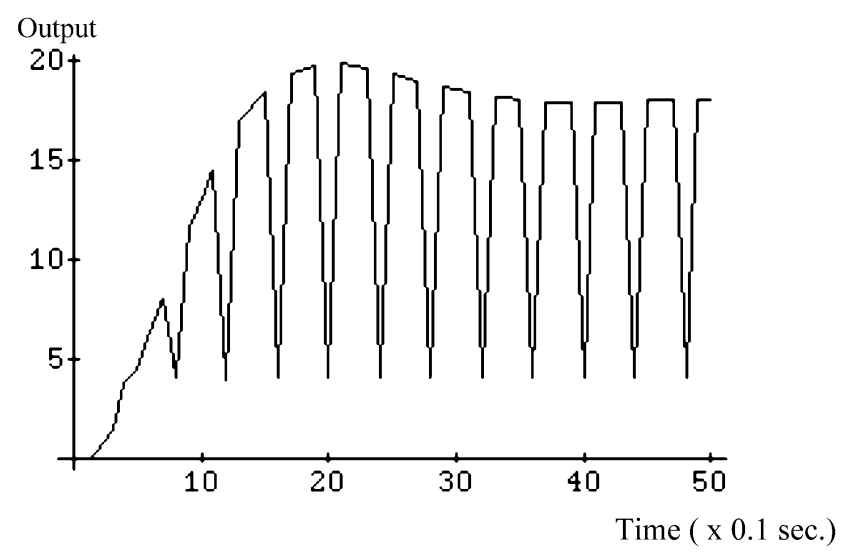

Fig. 1. Output versus time plot.

where $q$ is the discrete one-step advance operator and $D$ is the time-derivative operator. After substituting the control law in the plant description, the resulting closed-loop system is of the general form given while driven only by the disturbance $\delta(t)$. The signal $\delta(t)=\delta_{c}(t)$ is a perturbation which satisfies the general assumptions - constraints $C$ of the theory of total stability. There are six parameters to be estimated by the estimation schemes are $a_{1}=-1, a_{2}=2, a_{3}=3, b_{0}=1, b_{1}=b_{2}=2$ and $b_{3}=3$. The sampling period is $T=0.4$. Finally, the reference model is a third-order highly damped one of discrete regulation. The plant output is shown in Fig. 1.

Note that both the extended discrete system and the continuous one are stable since the output is bounded for all time. Remark 1, which is a combined interpretation of Propositions $1-3$, holds with all the signals in the loop being uniformly bounded for all time, i.e., "at" and "in-between" sampling instants. If the disturbance $\delta(t)$ is zeroed then the closed-loop system is globally asymptotically stable.

\section{Concluding Remarks}

The robust stability of a class of linear and time-varying hybrid systems has been investigated. The obtained sufficient conditions on robust stability are obtained by ensuring the total stability of the overall discretized system (whose state includes the digital sub-state plus the sampled continuous-time sub-state) at sampling instants and that of the continuous-time sub-state in-between sampling instants. The obtained sufficient conditions on total stability require the exponential stability of the uncoupled nominal continuous-time and digital subsystems, the sufficient weakness of the couplings between the linear parts of both sub-states and the sufficient smallness of the over-bounding functions of the state perturbations. Some links with previous work have been established for a special case of hybrid description through a combination of the obtained results with Schur's-type stability criteria for singularly perturbed systems. 


\section{References}

Bargelis, A., G. Hoehne and A. Cesnulevicus (2004). Intelligent functional model for costs minimization in hybrid manufacturing systems. Informatica, 15(1), 3-22.

Chaib, S., D. Boutat, A. Benali and J.P. Barbot (2005). Observability of the discrete state for dynamical piecewise hybrid systems. Nonlinear Analysis - Theory Methods \& Applications, 63(3), 423-438.

Hale, J.K. (1980). Ordinary Differential Equations. Wiley (Interscience), New York.

Kabamba, P.T., and S. Hara (1993). Worst-case analysis and design of sampled-data control systems. IEEE Trans. Automat. Contr., AC-38(9).

Kafri, W.S., and E.H. Abed (1996). Stability analysis of discrete-time singulary perturbed systems. IEEE Transactions on Circuits and Systems, $\mathbf{4 3}(10), 848-850$.

Kincaid, D., and W. Cheney (1991). Numerical Analysis. Brooks/Cole Publishing Co., Wadsworth Inc., Belmont, CA.

Kolev, L.V. (1993). Interval Methods for Circuit Analysis. World Scientific, Singapore.

Marchenko, V.M., and O.N. Poddubnaya (2005). Solution representations and relative controllability of linear differential algebraic systems with several delays. Doklady Mathematics, 72(2), 824-828.

Oppenheimer, E.P., and A.N. Michel (1988). Application of interval analysis techniques to linear systems: Part II - The interval matrix exponential function, and Part III - Initial value problems. IEEE Trans. Circuits and Systems, 35, 1230-1256.

De la Sen, M. (1996). The reachability and observability of hybrid multirate sampling linear systems. Computers and Mathematics Applic., 31(1), 109-122.

De la Sen, M. (2004). Sufficiency-type stability and stabilization criteria for linear time-invariant systems with constant point delays. Acta Applicandae Mathematicae, 83(3), 235-256.

De la Sen, M. (2006). On the adaptive control of a class of SISO dynamic hybrid systems. Applied Numerical Mathematics, 56(5), 618-647.

De la Sen, M., and C.F. Alastruey (2004). Stability results for two classes of linear time-delay and hybrid systems. Engineering Computations, 21(7), 718-735.

De la Sen, M., and Ningsu Luo (2004). On the uniform exponential stability of a wide class of linear time-delay systems. J. Math. Anal. Appl., 289(2), 456-476.

Shieh, L.S., J.S. Tsai and S.R. Lian (1986). Deterministic continuous-time state equations via the principal $q$ th root method. IEEE Trans. Automat. Contr., AC-31(5), 454-457. 
M. De la Sen was born in Arrigorriaga, Bizkaia in the Spanish Basque Country. He obtained the MSc degree with honors from the Basque Country University in 1975, the PhD degree in applied physics with high honors from the same University in 1979 and the degree of Docteur-d'Etat-és-Sciences Physiques (specialité Automatique et Traitement du Signal) from the Université de Grenoble, France with "mention trés honorable" in 1987. He has had several teaching and research positions in the University of the Basque Country in Bilbao (Spain) where he is currently professor of systems and control engineering in the Department of Systems and Control Engineering and head of the Institute of Research and Development of Processes IIDP. He has also had positions of visiting professor in the University of Grenoble (France), the University of Newcastle (New South Wales, Australia) and the Australian National University ANU (Canberra, Australia). He has been associate editor of the Electrosoft Journal (CML Mechanical and Computational Engineering publications) and he is currently associate editor of the journals Discrete Dynamics in Nature and Society (Hindawi Publishing Corporation), Applied Mathematical Sciences (Hikari Ltd.) and Nonlinear Analysis: Modelling and Control (Lithuanian Association of Nonlinear Analysts). He has been author or coauthor of a number of papers in the fields of of adaptive systems, mathematical systems theory, time-delay systems and ordinary differential equations, which are his main research interest subjects. He acts and has acted as reviewer for several international journals and conferences of control theory and engineering and applied mathematics. 


\section{Apie totalu vienos hibridiniu sistemu klasès stabiluma}

\section{Manuel De la SEN}

Taikant totalinio stabilumo teoremas, sukurtas tolydžiojo bei diskrečiojo laiko sistemoms, gauti patvariojo stabilumo rezultatai nominaliai tiesinèms hibridinèms sistemoms. Išnagrinèta hibridinių sistemų klasė, susidedanti iš tolydžiojo laiko ir skaitmeniniu sistemu junginio su perturbacijomis, kuriu nominalios (t.y. neperturbuotos) dalys, bendru atveju, esti tiesinės bei besikeičiančios laiko atžvilgiu. Gautos pakankamos patvariojo stabilumo sąlygos priklauso nuo parametrų verčių, apibrěžiančių neapibrèžtumų funkcijas bei analoginès ir skaitmeninès posistemių junginio taikymo pagrịstumą, numatant iš anksto, kad atitinkamos nesujungtos nominalios posistemès esti eksponentiškai stabilios. 